\title{
Mechanochemical Nucleophilic Substitution of Alcohols via Isouronium Intermediates
}

\author{
Tatsiana Dalidovich ${ }^{[\mathrm{a}]}$, Jagadeesh Varma Nallaparaju ${ }^{[\mathrm{a}]}$, Tatsiana Shalima ${ }^{[\mathrm{a}]}$, Prof. Dr. Riina Aav ${ }^{*[\mathrm{a}]}$ \\ and Dr. Dzmitry G. Kananovich*[a] \\ [a] Department of Chemistry and Biotechnology, Tallinn University of Technology, Akadeemia tee 15, 12618 Tallinn, \\ Estonia; E-mails: dzmitry.kananovich@taltech.ee, riina.aav@taltech.ee
}

\begin{abstract}
An expansion of the solvent-free synthetic toolbox is essential for advances in the sustainable chemical industry. Mechanochemical reactions offer a superior safety profile and reduced amount of waste compared to conventional solvent-based synthesis. Herein we report a new mechanochemical method for nucleophilic substitution of alcohols using fluoro- $N, N, N^{\prime}, N^{\prime}-$ tetramethylformamidinium hexafluorophosphate (TFFH) and $\mathrm{K}_{2} \mathrm{HPO}_{4}$ as an alcohol-activating reagent and a base, respectively. Alcohol activation and reaction with a nucleophile were performed in one milling jar via reactive isouronium intermediates. Nucleophilic substitution with amines afforded alkylated amines in 40-91\% yields. The complete stereoinversion occurred for the $\mathrm{S}_{\mathrm{N}} 2$ reaction of $(R)$ - and $(S)$-ethyl lactates. Substitution with halide anions $\left(\mathrm{F}^{-}, \mathrm{Br}^{-}, \mathrm{I}^{-}\right)$and oxygencentered $\left(\mathrm{CH}_{3} \mathrm{OH}, \mathrm{PhO}^{-}\right)$nucleophiles was also tested. Application of the method to the synthesis of active pharmaceutical ingredients (APIs) has been demonstrated.
\end{abstract}

\section{Introduction}

The synthesis of active pharmaceutical ingredients (APIs) driven by green chemistry concepts plays a pivotal role en route to a cleaner and safer pharmaceutical industry. ${ }^{1,2}$ Notably, a great deal of waste and safety concerns commonly originate from the use of solvents, typically responsible for 80-90\% of total mass consumption in a given industrial process. ${ }^{3}$ Besides environmental and safety issues, solvents can also generate dangerous contaminants in the produced APIs, such as cancerogenic nitrosamines derived from trace secondary amine impurities in $N, N$-dimethylformamide. ${ }^{4,5}$ In this regard, mechanochemistry ${ }^{6-8}$ as an essentially solvent-free technique can beneficially contribute to the ongoing green renovation of the pharma industry. ${ }^{9-11}$ Despite the rapid development of 
mechanochemical organic synthesis over the past decades, ${ }^{12-15}$ its synthetic portfolio still does not entirely cover the diverse range of transformations required for API production and needs to be expanded.

Nitrogen-containing functional groups prevail in the existing immense number of bioactive molecules, including APIs. ${ }^{16}$ As a consequence, almost $80 \%$ of heteroatom alkylation and arylation reactions used in the synthesis of drug candidates involve the formation of $\mathrm{C}-\mathrm{N}$ bonds. ${ }^{17}$ In addition to the mainstream developments like amide synthesis, ${ }^{18-23}$ the state-of-the-art mechanochemical synthetic toolbox offers several opportunities to construct $\mathrm{C}-\mathrm{N}$ bonds ${ }^{24}$ in amines via alkylation ${ }^{25-27}$ and arylation ${ }^{28-30}$ with organic halides. However, the use of alcohols as ubiquitous starting materials remains nearly untapped. ${ }^{31}$ Nucleophilic substitution of the hydroxyl group in alcohols is one of the most fundamental and widespread chemical transformations. Applied to amine synthesis, alcohols can serve as safer replacements to lacrimatic and potentially genotoxic organic halides. However, the direct nucleophilic substitution of alcohols remains a challenge, ${ }^{2,32-36}$ and prior transformation of hydroxyl into a better leaving group (e.g., sulfonate ester) is commonly required.

Recently, our group reported a new mechanochemical amidation approach based on the use of reactive uronium-type amide coupling reagents such as (1-cyano-2-ethoxy-2oxoethylidenaminooxy)dimethylaminomorpholinocarbenium hexafluorophosphate (COMU) or $N, N, N^{\prime}, N^{\prime}$-tetramethylchloroformamidinium hexafluorophosphate $(\mathrm{TCFH})$ and $\mathrm{K}_{2} \mathrm{HPO}_{4}$ as a base (Scheme 1, A). ${ }^{37}$ The reaction proceeded via the generation of activated derivatives of carboxylic acids, such as acyl uroniums and acyl phosphates.

Inspired by these findings, we planned to extend the same activation methodology towards nucleophilic substitution of alcohols (Scheme 1, B). Indeed, $O$-alkyl isouronium salts can be obtained by the reactions of alcohols with halouronium salts (e.g., TCFH or its fluoro-analog TFFH), a process commonly utilized for the preparation of amide coupling reagents. ${ }^{38,39}$ Similarly to $O$-alkyl isoureas generated from alcohols and carbodiimides, ${ }^{40-44}$ isouronium salts can act as reactive intermediates in nucleophilic displacement reactions. ${ }^{45,46}$ However, none of these alcohol-activation techniques have been previously attempted under solvent-free conditions or utilized for the synthesis of amines. Here we report for the first time the application of an air-stable and non-hygroscopic solid TFFH reagent ${ }^{46-}$ ${ }^{48}$ for activation of alcohols towards nucleophilic substitution with amines, halogens and some oxygen nucleophiles under essentially solvent-free conditions provided by mechanochemistry. 

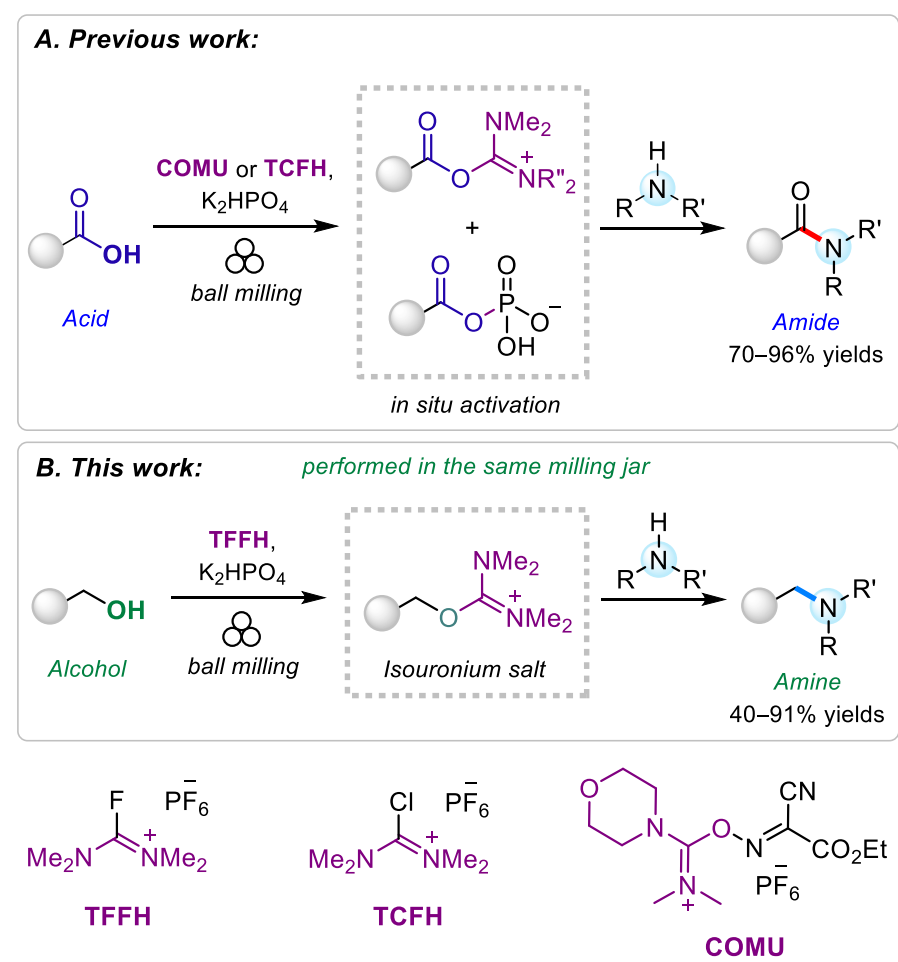

Scheme 1. Mechanochemical synthesis of amides via in situ activation of carboxylic acids ${ }^{37}$ and preparation of amines from alcohols.

\section{Results and Discussion}

Optimization of coupling of alcohols with amines. The reaction between 1-naphthalenemethanol (1) and morpholine as a nitrogen nucleophile was selected as a model transformation to find the optimal reaction conditions leading to tertiary amine product 2 (Scheme 2). Ball milling experiments were performed in a Form-Tech Scientific FTS1000 shaker mill operating at $30 \mathrm{~Hz}$ using a $14 \mathrm{~mL}$ zirconia-coated milling jar and a single $10 \mathrm{~mm}$ milling ball. In contrast to amide synthesis, ${ }^{37}$ attempted in situ activation of alcohol 1 with TCFH and TFFH reagents in the presence of morpholine resulted in a very low yield of 2 (less than 8\%; see Table S1 in the Supporting Information). This result is a likely outcome of a faster competitive reaction of halouronium reagents with morpholine itself. $^{48,49}$ To overcome the problem, a stepwise reaction in a single milling jar was considered, which involved the generation of isouronium salt $\mathbf{3}$ by the reaction of $\mathbf{1}$ with a halouronuim reagent before adding morpholine. To our delight, this technique afforded high 88-91\% conversions to 3 by milling alcohol 1 with TFFH reagent in the presence of $\mathrm{K}_{2} \mathrm{HPO}_{4}$ as a base for $1 \mathrm{~h}$ (Scheme 2). The addition of morpholine to the same reaction jar resulted in fast (less than $20 \mathrm{~min}$ ) and quantitative transformation of $\mathbf{3}$ into amine $\mathbf{2}$, which was isolated in $82 \%$ yield. The same reaction sequence 
performed in acetonitrile solution with trimethylamine as a base (see Table S5 in the Supporting Information) produced 2 in noticeably lower yield (did not exceed 50\% according to ${ }^{1} \mathrm{H}$ NMR analysis).
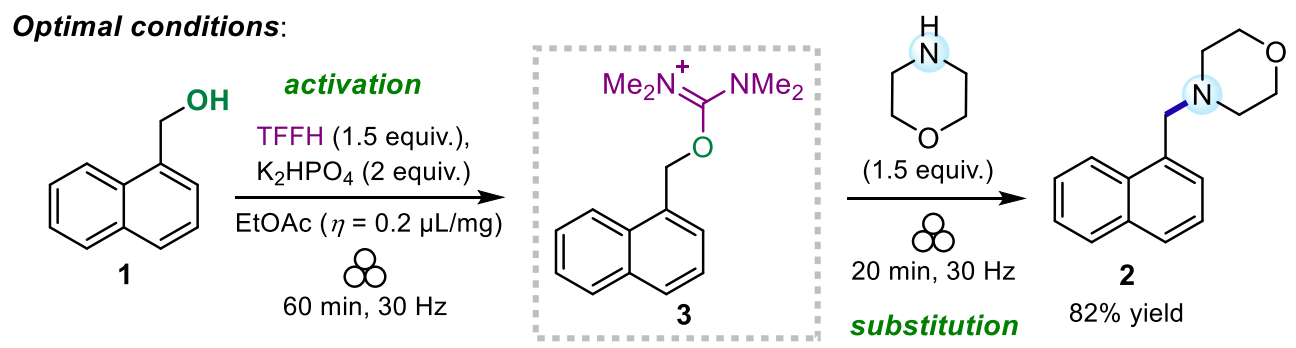

$88-91 \%\left({ }^{1} \mathrm{H}\right.$ NMR $)$

Deviation from the optimal conditions:
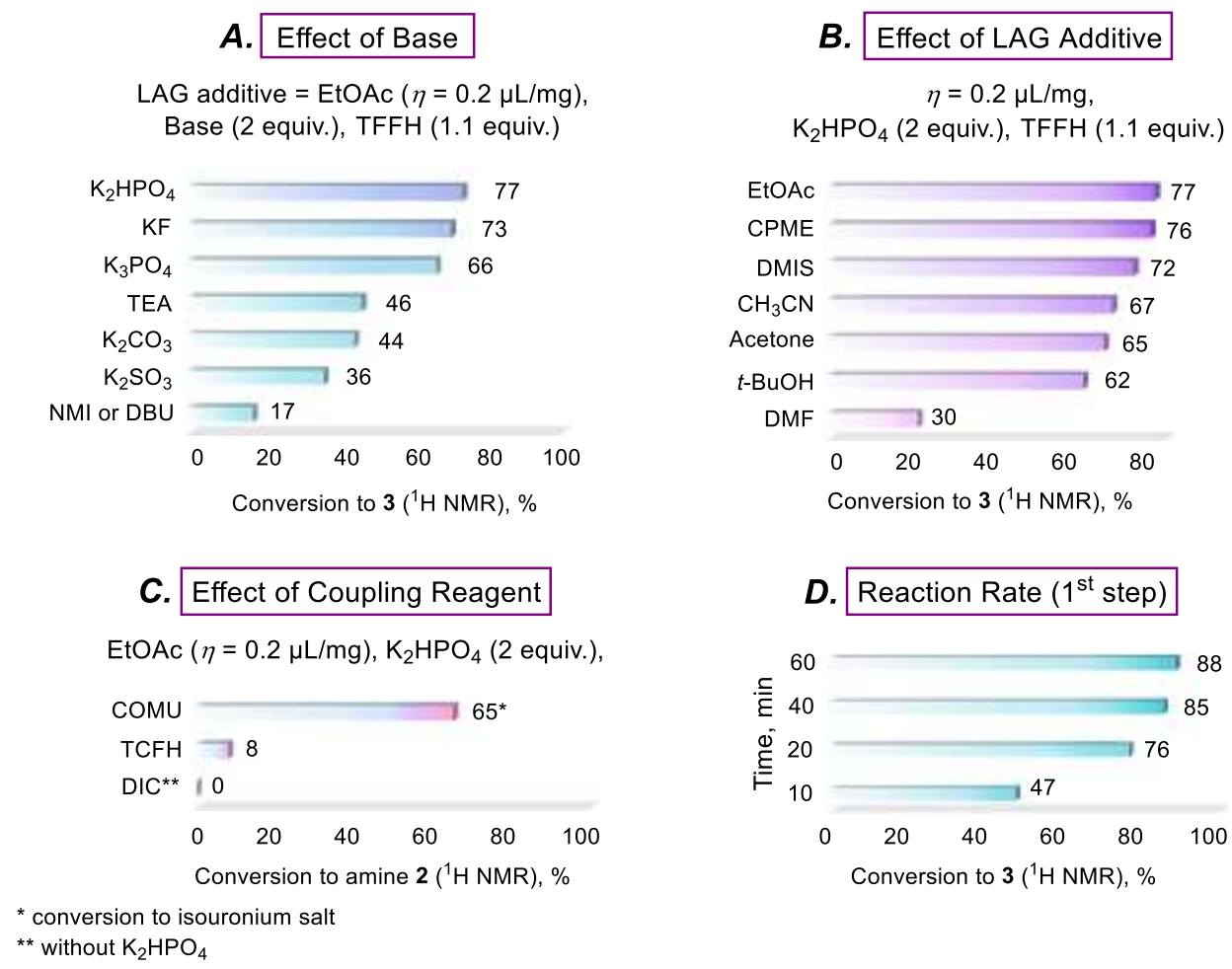

** without $\mathrm{K}_{2} \mathrm{HPO}_{4}$

Scheme 2. Selected optimization experiments (performed with $0.16 \mathrm{mmol}$ of alcohol 1 as a limiting substrate) and kinetic studies. TEA $=$ trimethylamine, $\mathrm{NMI}=N$-methylimidazole, $\mathrm{DBU}=1,8$ diazabicyclo[5.4.0] undec-7-ene, $\mathrm{DIC}=N, N^{\prime}$-diisopropylcarbodiimide, $\mathrm{CPME}=$ cyclopentyl methyl ether; DMIS = dimethyl isosorbide. The complete dataset of optimization experiments is provided in the Supporting Information (Table S2).

Selection of base, liquid additive for liquid-assisted grinding (LAG) ${ }^{6,7}$ and coupling reagent were identified as a trio of most crucial parameters affecting the reaction efficiency. No reaction of $\mathbf{1}$ and TFFH occurred without a basic additive. Concerning the choice of base (Scheme 2, A), replacement of organic bases like triethylamine (TEA), $N$-methylimidazole (NMI) and diazabicyclo(5.4.0)undec- 
7-ene (DBU) with inexpensive and less hazardous inorganic salts was superior (e.g., $\mathrm{K}_{2} \mathrm{HPO}_{4}$ or $\left.\mathrm{K}_{3} \mathrm{PO}_{4}\right)$ or at least as efficient $\left(\mathrm{K}_{2} \mathrm{CO}_{3} v s\right.$. TEA). Potassium fluoride (KF) and dipotassium phosphate $\left(\mathrm{K}_{2} \mathrm{HPO}_{4}\right)$ were identified as the most efficient bases during the initial screening, affording $73 \%$ and $77 \%$ conversions to 3 , respectively. In the case of $\mathrm{K}_{2} \mathrm{HPO}_{4}$, a slight increase of TFFH amount (1.5 equiv.) to compensate for pyrophosphate formation (see the Supporting Information, Fig. S2) delivered the highest conversion values (88-91\%).

A minor amount of liquid additive usually accelerates mechanochemical reactions, a technique known as liquid-assisted grinding (LAG). It is empirically characterized by the parameter $\eta$, expressing the ratio of the volume of liquid $(\mu \mathrm{L})$ added to the total amount of solid $(\mathrm{mg}) .{ }^{50}$ Although LAG requires a subtle amount of liquid (fixed at $0.2 \mu \mathrm{L} / \mathrm{mg}$ in our experiments), we preferred to screen several green solvents as LAG additives, ${ }^{51,52}$ in addition to several conventional polar solvents (Scheme 2, B; see Table S2 in the Supporting Information for additional data). In our hands, green solvents like ethyl acetate (EtOAc), cyclopentyl methyl ether (CPME) and dimethyl isosorbide (DMIS) served as excellent LAG additives offering the best conversions in the $72-77 \%$ range and outperforming other polar solvents.

Among the activating reagents, TFFH was identified as the best activator, producing isouronium salt 3 in the highest yield. In comparison, COMU delivered lower $65 \%$ yield of the corresponding isouronium intermediate (Scheme 2, C). In contrast to TFFH, the corresponding chlorine reagent TCFH was found almost unreactive towards alcohol 1, with the same order of reactivity also observed in acetonitrile solution (see Table S5 in the Supporting Information). The use of TFFH is also preferable to TCFH or COMU in view of the lowest molecular weight and, therefore, better atom economy for the former. The reaction of alcohol 1 with $N, N^{\prime}$-diisopropylcarbodiimide (DIC) led to the formation of the corresponding $O$-alkyl isourea ${ }^{42}$ but did not result in any further nucleophilic substitution reaction (see Table S2 in the Supporting Information for the details). Note that at least 60 min milling time was required to achieve the highest conversion (88-91\%) of starting alcohol 1 into intermediate $\mathbf{3}$, as a kinetic study revealed (Scheme 2, D).

Scope and Limitations. With the optimal conditions established, the substrate scope of various alcohols was examined (Scheme 3). In addition to the above-mentioned amine 2 , analogous morpholine derivatives 4-6 were flawlessly prepared in 61-91\% yields from the corresponding benzylic-type primary alcohols. Substitution of the aromatic ring with electron-donating (OMe) or electron-withdrawing $\left(\mathrm{CF}_{3}\right)$ groups did not substantially affect the reaction efficacy (5 and $\mathbf{6}$, Scheme 3). 


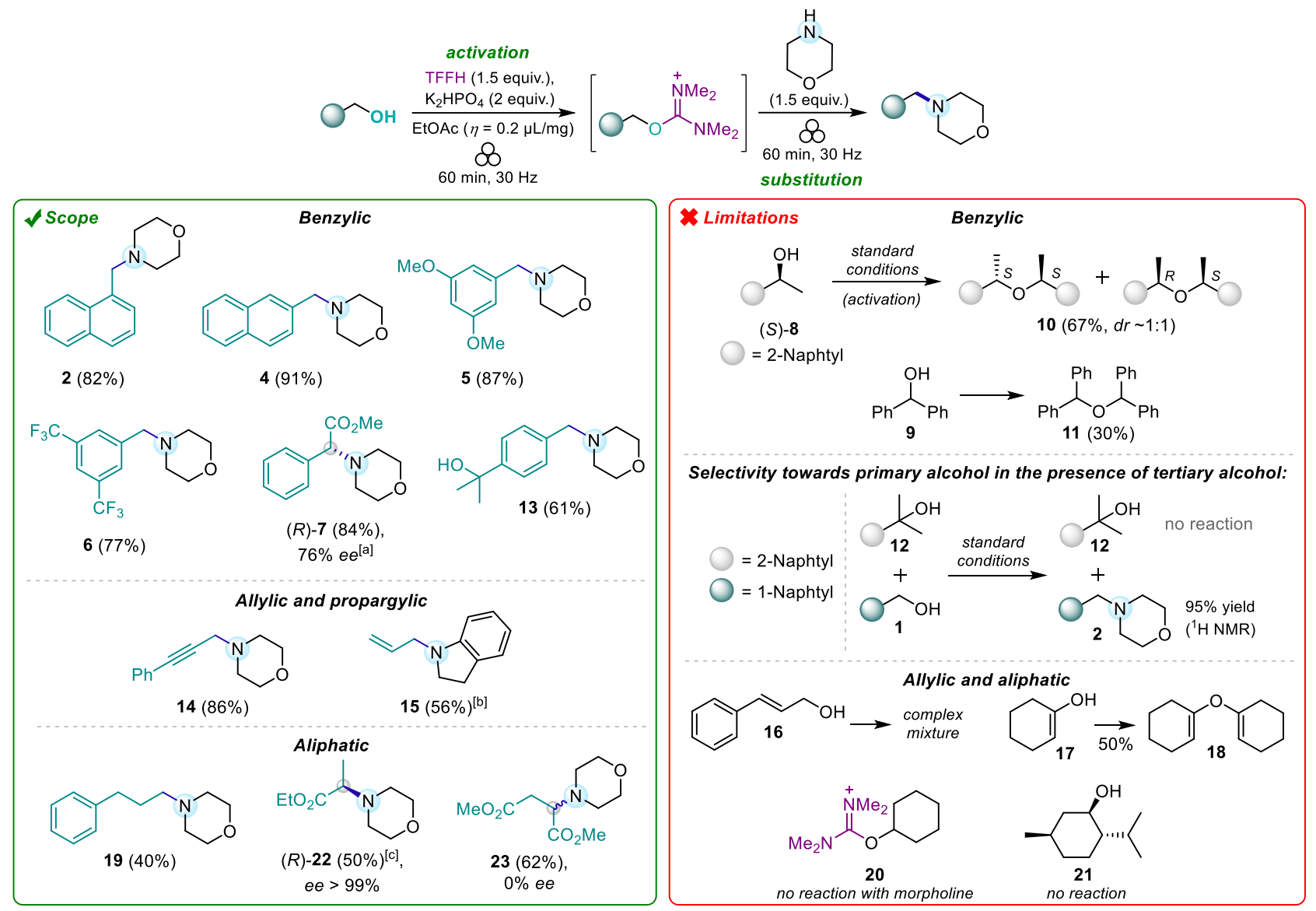

Scheme 3. Scope of alcohols and limitations of amine synthesis (performed with $0.25-0.60 \mathrm{mmol}$ of alcohols as limiting substrates). ${ }^{[\mathrm{a}]} 85 \%$ ee after 15 min milling with TFFH/K ${ }_{2} \mathrm{HPO}_{4}{ }^{\left[{ }^{[\mathrm{b}]} \text { Indoline was }\right.}$ used instead of morpholine. ${ }^{[\mathrm{c}]}$ Milling time with morpholine $3 \mathrm{~h}$. $(S)$-22 was prepared from $(R)$-lactate with the same yield and optical purity.

Coming to secondary benzylic substrates, $(R)$-phenylglycine derivative 7 was obtained from optically pure (> 99\% ee) methyl (S)-mandelate in high 84\% yield, but with reduced optical purity (76\% ee). Although phenylglycine is known as one of the most epimerization-prone amino acids, ${ }^{53}$ we found that optical purity of 7 remained almost unchanged over variation of milling time with morpholine (30-120 min; see Table S6 in the Supporting Information). However, shortening the duration of the alcohol activation step to $15 \mathrm{~min}$ resulted in a noticeably better $85 \%$ ee. These results indicate that partial epimerization of the corresponding isouronium intermediate could occur (presumably via enolization ${ }^{54}$ ) while its $S_{N} 2$ reaction with the amine is faster and dominant. At the same time, secondary benzylic alcohols like $(S)$ - $\alpha$-methyl-2-naphthalenemethanol (8) and diphenylmethanol (9) produced ethers 10 and 11 during the milling with $\mathrm{TFFH} / \mathrm{K}_{2} \mathrm{HPO}_{4}$. Ether 10 was obtained as an almost equimolecular mixture of $(S, S)$ - and $(R, S)$-diastereomers according to ${ }^{1} \mathrm{H}$ NMR and HPLC analysis on a chiral stationary phase, indicating that the reaction followed the $\mathrm{S}_{\mathrm{N}} 1$ mechanism and occurred 
via the corresponding benzylic cation. Tertiary benzylic alcohol 12 was unreactive and did not form the corresponding isouronium salt. Notably, highly chemoselective amination of primary alcohol 1 was achieved in the presence of tertiary substrate $\mathbf{1 2}$ due to such a high difference in reactivity. This finding was applied in the preparation of amine 13, which had an intact tertiary alcoholic moiety. Amines 14 and 15 were successfully prepared from the corresponding propargylic and allylic alcohols in 86\% and 56\% yields, respectively. However, other tested allylic substrates like alcohols 16 and 17 failed to render the corresponding amine products due to several dominant side processes, such as ether formation. Under the standard reaction conditions, 3-phenylpropyl amine 19 was produced from the corresponding alcoholic precursor in a low $40 \%$ yield, evidently due to the reduced reactivity of the corresponding isouronium salt with an aliphatic side chain. The yield of $\mathbf{1 9}$ was not significantly improved even after extended milling time with an amine $(3 \mathrm{~h})$. Secondary aliphatic alcohols either produced unreactive isouronium salts (e.g., 20 obtained from cyclohexanol) or did not react at all (e.g., menthol 21). Intriguingly, ethyl $(S)$ - and $(R)$-lactates were quite reactive under the developed conditions, producing alanine derivative 22 in $50 \%$ yield and excellent optical purity (> 99\% ee). The stereochemical outcome indicates complete stereoinversion via the $S_{N} 2$ mechanism. Surprisingly, a derivative of aspartic acid $\mathbf{2 3}$ was obtained from dimethyl $(S)$-malate with a total loss of optical purity. This result, along with a substantial amount of dimethyl fumarate detected in the reaction mixture (see Figure S6 in the Supporting Information), indicates that substitution of hydroxyl probably occurred via an elimination-addition mechanism in this case.

The scope of amines was explored in the substitution reactions of 2-naphthylmethanol 24 (Scheme 4). Secondary aliphatic amines like thiomorpholine, piperidine and the methyl ester of D-proline delivered corresponding amines $\mathbf{2 5}-\mathbf{2 7}$ in moderate to high yields (42-79\%). Notably, less nucleophilic aromatic amine (indoline) delivered a high $86 \%$ yield of the corresponding amine product 28. Primary amines, for example, 3,5-bis(trifluoromethyl)benzylamine (29), were found reacting stepwise under the standard reaction conditions, producing a mixture of mono- and dialkylated products $\mathbf{3 0}$ and $\mathbf{3 1}$, respectively. Full conversion into the tertiary amine product can be achieved using stoichiometric amounts (0.5 equiv.) of a primary amine; for example, amine $\mathbf{3 2}$ was prepared from benzylamine in $77 \%$ yield under these conditions. 


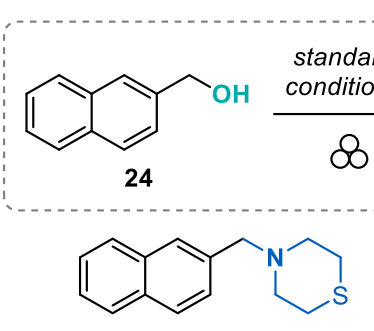

$25(79 \%)$

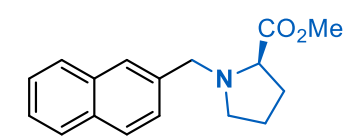

$27(42 \%)^{[a]}$

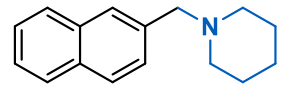

$26(52 \%)$

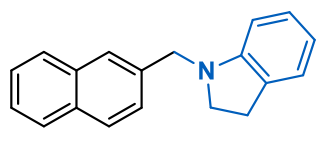

$28(86 \%)$

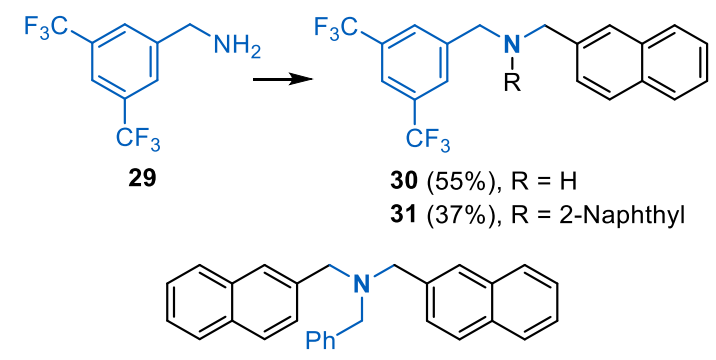

$32(77 \%)^{[b]}$

Scheme 4. Scope of amines 25-32 prepared from 2-naphthalenemethanol (24). (Performed with 0.25 mmol of $\mathbf{2 4}$ as a limiting substrate). ${ }^{\text {[a] }}$ Amine hydrochloride was used as starting material. ${ }^{[b]}$ With 0.5 equiv. of benzylamine.

Application to the synthesis of APIs and bioactive amines. The knowledge acquired during the optimization studies and examination of substrate scope prompted us to implement the synthesis of several bioactive amines (including APIs) using mechanochemical $\mathrm{C}-\mathrm{N}$ bond-forming reactions as the key steps. Thus, a stimulant drug 1-methyl-4-benzylpiperazine (33, MBZP) was prepared by alkylation of $N$-methylpiperazine (34) with benzylic alcohol and isolated in $54 \%$ yield by crystallization of its hydrochloric salt from methanol (Scheme 5). Antidepressant piberaline (35) was prepared from picolinic acid (36) via stepwise mechanochemical amide coupling, ${ }^{37}$ solvent-free Bocdeprotection $^{55}$ and mechanochemical amination steps. Derivatives of 4-(hydroxymethyl)benzoic acid (39) have multiple pharmaceutical uses, including anticancer agents (e.g., Imanitib ${ }^{56}$ Masitinib $^{57}$ ), histone deacetylase inhibitors, ${ }^{58}$ histamine receptor antagonists (e.g., Bavisant). ${ }^{59}$ The observed low reactivity of TCFH reagent towards alcohols compared to TFFH (see Scheme 2, C) and, conversely, the high amide coupling potency of the $\mathrm{TCFH} / \mathrm{K}_{2} \mathrm{HPO}_{4}$ reagent system ${ }^{37}$ was utilized to perform protecting group-free mechanochemical amidation of carboxylic group in 39 (Scheme 6) while leaving the benzylic hydroxyl intact. Switching to the TFFH reagent at the next step resulted in activation of benzylic alcohol $\mathbf{4 0}$ and yielded the morpholine derivative ${ }^{59} \mathbf{4 1}$ in $80 \%$ yield. 


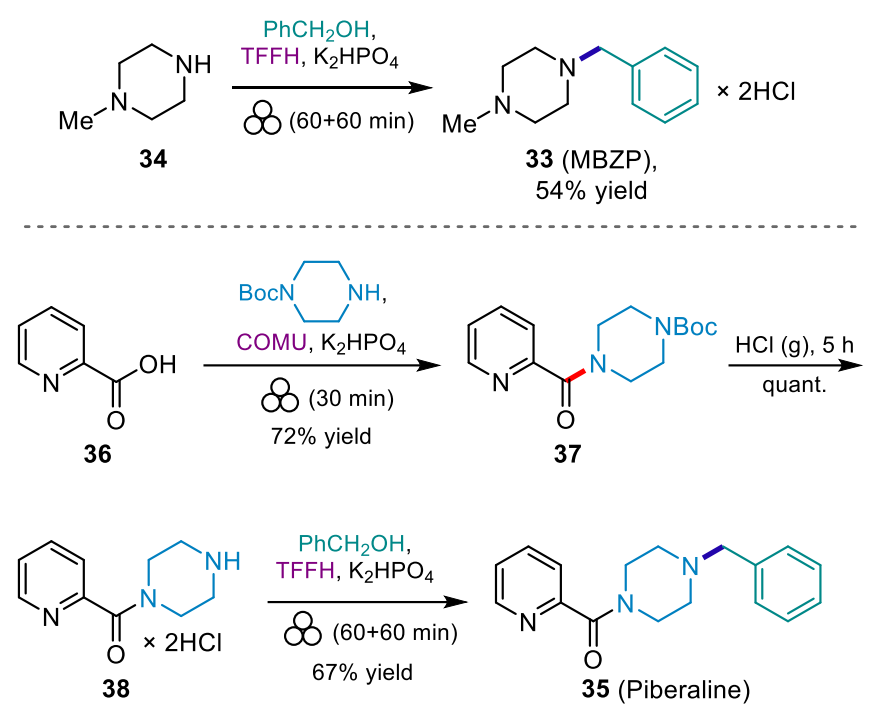

Scheme 5. Mechanochemical $\mathrm{C}-\mathrm{N}$ bond-forming reactions in the synthesis of antidepressants 1-methyl-4-benzylpiperazine (33, MBZP) and piberaline (35).
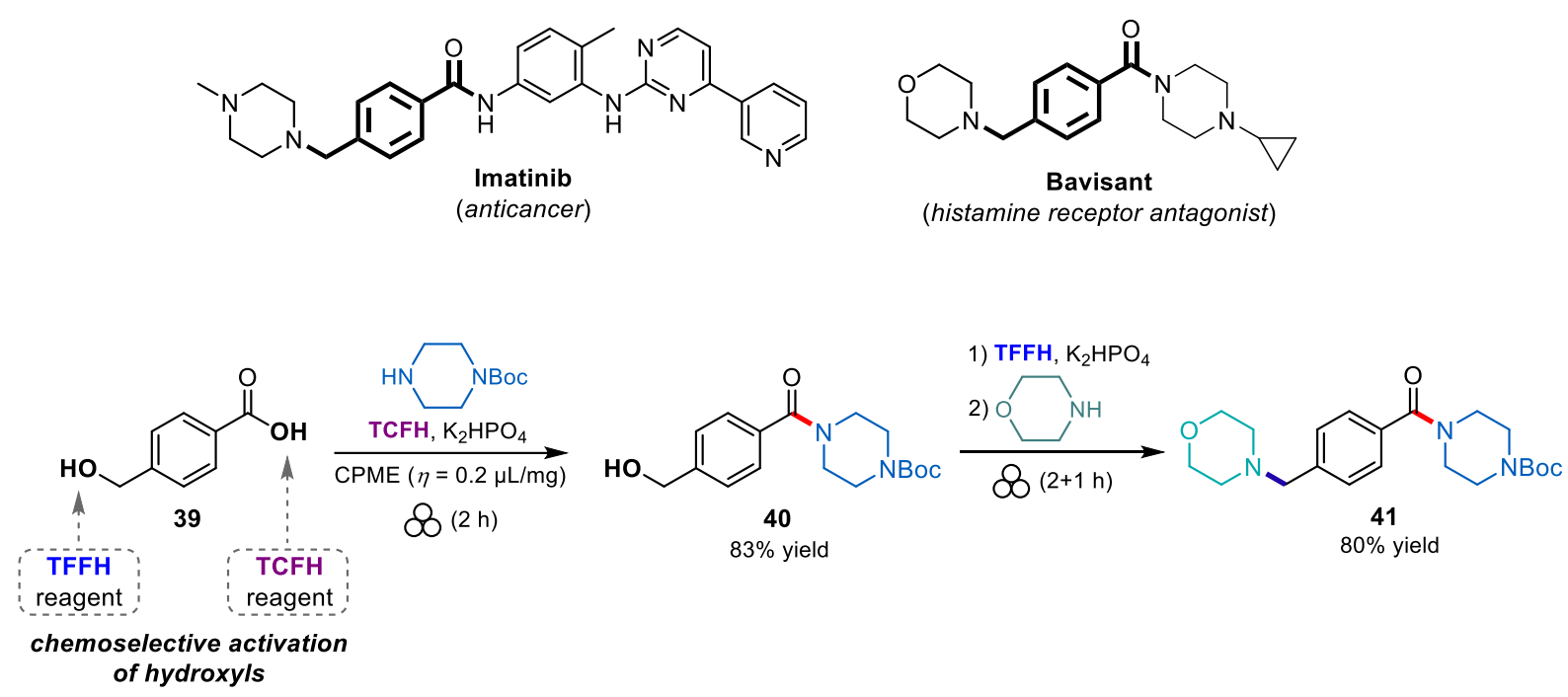

Scheme 6. Examples of pharmacologically active derivatives of 4-(hydroxymethyl)benzoic acid (39) and preparation of amine $\mathbf{4 1}$ via chemoselective activation of hydroxyls in $\mathbf{3 9}$.

Nucleophilic substitution with halogens and oxygen nucleophiles. In addition to amines and their derivatives, oxygen- and halogen-containing moieties occupy a spacious fraction of pharmaceutically relevant functional groups. ${ }^{16}$ This fact inspired us to examine the efficacy of the developed $\mathrm{OH}$ activation protocol towards incorporating halogens and oxygen-containing functionalities. The corresponding nucleophilic substitution reactions were performed with 1- and 2naphthalenemethanols (1 and 24, Scheme 7). Activation of the hydroxyl in $\mathbf{2 4}$ with the TFFH reagent followed by milling the produced isouronium intermediate with solid $\mathrm{MgBr}_{2}$ or $\mathrm{KI}$ afforded the 
corresponding bromo and iodo derivatives in high yields (42 and 43; Eq. 1 and 2). Notably, no fluoro derivative was produced during the milling with TFFH/KF, both serving as a source of a fluoride anion. Only replacing KF with $\mathrm{CsF}$ resulted in the generation of fluorine derivative 44 (Eq. 3), although in a moderate $40 \%$ yield. This result stays in line with previous observations, ${ }^{60,61}$ showing that nucleophilic reactivity of anions under solvent-free conditions is strongly controlled by their counter-ion pairing. The effect was interpreted in the framework of hard-soft acid-base (HSAB) theory, ${ }^{14}$ explaining the enhanced nucleophilicity of $\mathrm{F}^{-}$in $\mathrm{CsF}$ as a result of a mismatched soft-hard ion pair. Higher nucleophilic reactivity of $\mathrm{F}^{-}$in $\mathrm{CsF}$ compared to $\mathrm{KF}$ also correlates with lower lattice energy for the former $\left(\mathrm{CsF}, 740 \mathrm{~kJ} \cdot \mathrm{mol}^{-1}\right.$; KF, $\left.821 \mathrm{~kJ} \mathrm{~mol}{ }^{-1}\right){ }^{62}$ Use of potassium phenolate as the nucleophile resulted in a modest $46 \%$ yield of the corresponding substitution product 45 (Eq. 4). At the same time, the isouronium salt generated from 2-naphthalenemethanol was highly sensitive towards solvolysis in a nucleophilic solvent such as methanol and produced methyl ether 46 in high 91\% yield (Eq. 5) after $3 \mathrm{~h}$.

(1)

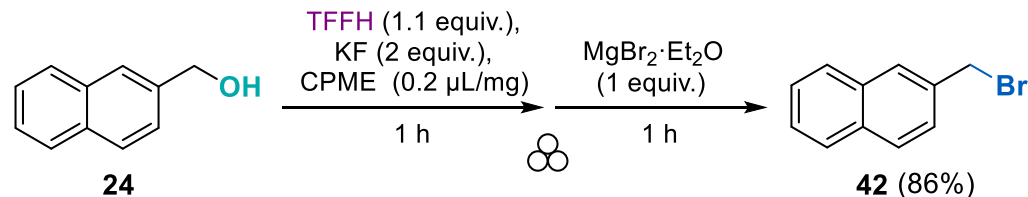

(2)

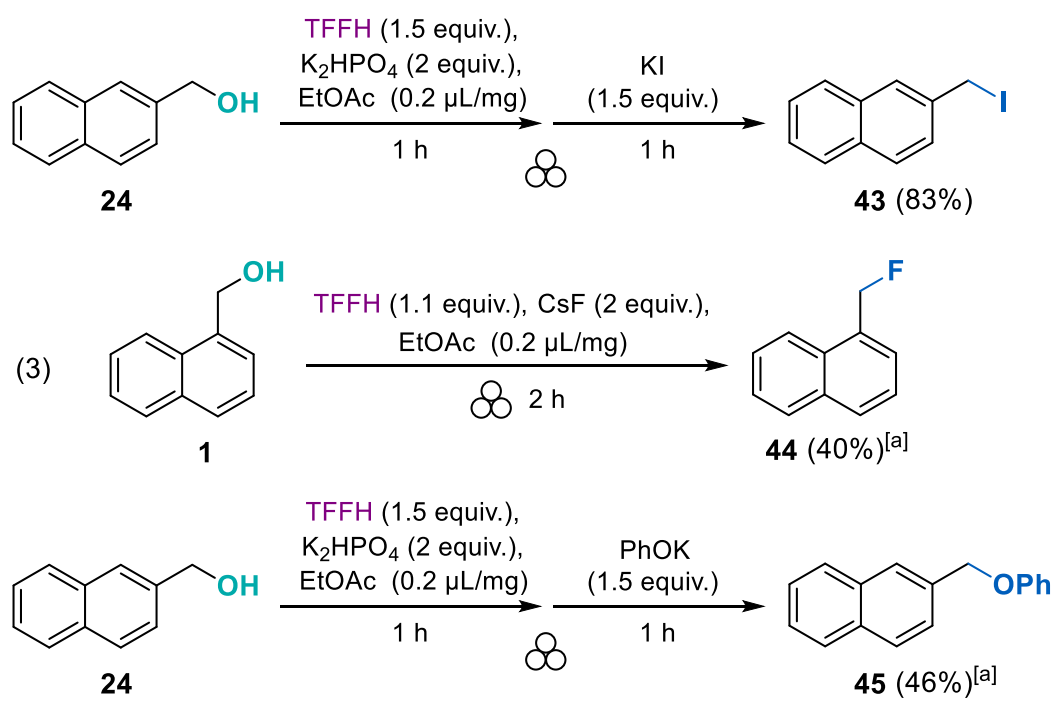

(5)

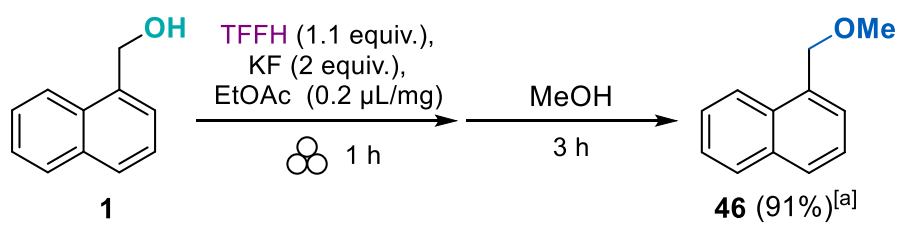

Scheme 7. The substitution reactions of naphthalenemethanols with various nucleophiles. ${ }^{\text {a] }}{ }^{1} \mathrm{H}$ NMR yield. 


\section{Conclusion}

In conclusion, we have developed a new mechanochemical protocol for nucleophilic substitution of alcohols by applying TFFH as an activator and $\mathrm{K}_{2} \mathrm{HPO}_{4}$ as a non-toxic inorganic base. This combination of reagents enables fast (within $1 \mathrm{~h}$ ) and effective conversion of alcohols into reactive $O$-alkyl isouronium salts under ball milling conditions with sustainable solvents (EtOAc, CPME) as LAG additives. We showed for the first time that $O$-alkyl isouronium salts could act as highly reactive activated derivatives of alcohols in nucleophilic displacement reactions with amines, under mechanochemical conditions or in solution. Thus, mechanochemically generated isouronium intermediates afforded a range of $N$-alkylated amine products in 40-91\% yield after ball milling with amines in the same reaction vessel. Complete stereoinversion was attained in the substitution reactions of ethyl $(S)$ - and $(R)$-lactates with morpholine, while enolizable $\alpha$-hydroxy esters could result in reduced or even fully demolished optical purity of the product. The developed substitution protocol is particularly efficient for reactive alcoholic substrates like primary benzylic alcohols. However, steric hindrances (tertiary, secondary substrates) or reduced reactivity in nucleophilic displacement reactions (aliphatic substrates) could substantially reduce the yield of amine products or even prevent the reaction from occurring. These limitations were advantageous when performing chemoselective transformations, such as substituting a primary benzylic alcohol in the presence of a tertiary alcoholic function. A striking difference in the chemoselectivity of fluoro- and chlorouronium

coupling reagents (TFFH vs. TCFH) allowed performing amide coupling of 4(hydroxymethyl)benzoic acid with an unprotected benzylic alcohol moiety. The developed process expands the synthetic portfolio of mechanochemical reactions. It can serve as a beneficial supplement to the existing $\mathrm{C}-\mathrm{N}$ bond-forming protocols, which are suitable for multistep mechanosynthesis of APIs with a decreased environmental impact and a better safety profile.

\section{Experimental Section}

Detailed information about optimization studies, experimental methods and the corresponding structure characterization data are given in the Supporting Information.

\section{Acknowledgements}

The research was supported by Estonian Research Council grant PRG399 and support from COST Action CA18112 "Mechanochemistry for Sustainable Industry”, the ERDF CoE in Molecular Cell 
Engineering 2014-2020.4.01.15-0013, EU Regional Development Fund and Dora Plus Program are gratefully acknowledged. We are also grateful to Dr. Alexander-Mati Müürisepp (TalTech) for measuring IR spectra.

\title{
Conflict of interest
}

The authors declare no conflict of interest.

\author{
Keywords: mechanochemistry $\bullet$ solvent-free chemistry $\bullet$ amines $\bullet$ isouronium $\bullet$ alcohols • \\ pharmaceuticals • nucleophilic substitution
}

\section{References}

(1) Constable, D. J. C.; Dunn, P. J.; Hayler, J. D.; Humphrey, G. R.; Leazer, Jr., J. L.; Linderman, R. J.; Lorenz, K.; Manley, J.; Pearlman, B. A.; Wells, A.; Zaks, A.; Zhang, T. Y. Key Green Chemistry Research Areas-a Perspective from Pharmaceutical Manufacturers. Green Chem. 2007, 9 (5), 411-420. https://doi.org/10.1039/B703488C.

(2) Bryan, M. C.; Dunn, P. J.; Entwistle, D.; Gallou, F.; Koenig, S. G.; Hayler, J. D.; Hickey, M. R.; Hughes, S.; Kopach, M. E.; Moine, G.; Richardson, P.; Roschangar, F.; Steven, A.; Weiberth, F. J. Key Green Chemistry Research Areas from a Pharmaceutical Manufacturers' Perspective Revisited. Green Chem. 2018, 20 (22), 5082-5103. https://doi.org/10.1039/C8GC01276H.

(3) Constable, D. J. C.; Jimenez-Gonzalez, C.; Henderson, R. K. Perspective on Solvent Use in the Pharmaceutical Industry. Org. Process Res. Dev. 2007, 11 (1), 133-137. https://doi.org/10.1021/op060170h.

(4) López-Rodríguez, R.; McManus, J. A.; Murphy, N. S.; Ott, M. A.; Burns, M. J. Pathways for N-Nitroso Compound Formation: Secondary Amines and Beyond. Org. Process Res. Dev. 2020, 24 (9), 1558-1585. https://doi.org/10.1021/acs.oprd.0c00323.

(5) Sörgel, F.; Kinzig, M.; Abdel-Tawab, M.; Bidmon, C.; Schreiber, A.; Ermel, S.; Wohlfart, J.; Besa, A.; Scherf-Clavel, O.; Holzgrabe, U. The Contamination of Valsartan and Other Sartans, Part 1: New Findings. J. Pharm. Biomed. Anal. 2019, 172, 395-405. https://doi.org/https://doi.org/10.1016/j.jpba.2019.05.022.

(6) Do, J.-L.; Friščić, T. Mechanochemistry: A Force of Synthesis. ACS Cent. Sci. 2017, 3 (1), 13-19. https://doi.org/10.1021/acscentsci.6b00277.

(7) Do, J. L.; Friščić, T. Chemistry 20: Developing a New, Solvent-Free System of Chemical Synthesis Based on Mechanochemistry. Synlett 2017, 28 (16), 2066-2092. https://doi.org/10.1055/s-0036-1590854.

(8) James, S. L.; Adams, C. J.; Bolm, C.; Braga, D.; Collier, P.; Friščić, T.; Grepioni, F.; Harris, K. D. M.; Hyett, G.; Jones, W.; Krebs, A.; Mack, J.; Maini, L.; Orpen, A. G.; Parkin, I. P.; Shearouse, W. C.; Steed, J. W.; Waddell, D. C. Mechanochemistry: Opportunities for New and Cleaner Synthesis. Chem. Soc. Rev. 2012, 41 (1), 413-447. https://doi.org/10.1039/C1CS15171A.

(9) Tan, D.; Loots, L.; Friščić, T. Towards Medicinal Mechanochemistry: Evolution of Milling from Pharmaceutical Solid Form Screening to the Synthesis of Active Pharmaceutical Ingredients (APIs). Chem. Commun. 2016, 52 (50), 7760-7781. https://doi.org/10.1039/C6CC02015A.

(10) Colacino, E.; Porcheddu, A.; Charnay, C.; Delogu, F. From Enabling Technologies to Medicinal Mechanochemistry: An Eco-Friendly Access to Hydantoin-Based Active Pharmaceutical Ingredients. React. Chem. Eng. 2019, 4 (7), 1179-1188. https://doi.org/10.1039/C9RE00069K.

(11) Ying, P.; Yu, J.; Su, W. Liquid-Assisted Grinding Mechanochemistry in the Synthesis of Pharmaceuticals. Adv. Synth. Catal. 2021, 363 (5), 12461271. https://doi.org/https://doi.org/10.1002/adsc.202001245.

(12) Howard, J. L.; Cao, Q.; Browne, D. L. Mechanochemistry as an Emerging Tool for Molecular Synthesis: What Can It Offer? Chem. Sci. 2018, 9 (12), 3080-3094. https://doi.org/10.1039/C7SC05371A.

(13) Tan, D.; Friščić, T. Mechanochemistry for Organic Chemists: An Update. European J. Org. Chem. 2018, 2018 (1), $18-33$. https://doi.org/https://doi.org/10.1002/ejoc.201700961. 
(14) Andersen, J.; Mack, J. Mechanochemistry and Organic Synthesis: From Mystical to Practical. Green Chem. 2018, 20 (7), $1435-1443$. https://doi.org/10.1039/c7gc03797j.

(15) Porcheddu, A.; Colacino, E.; De Luca, L.; Delogu, F. Metal-Mediated and Metal-Catalyzed Reactions Under Mechanochemical Conditions. ACS Catal. 2020, 10 (15), 8344-8394. https://doi.org/10.1021/acscatal.0c00142.

(16) Ertl, P.; Altmann, E.; McKenna, J. M. The Most Common Functional Groups in Bioactive Molecules and How Their Popularity Has Evolved over Time. J. Med. Chem. 2020, 63 (15), 8408-8418. https://doi.org/10.1021/acs.jmedchem.0c00754.

(17) Roughley, S. D.; Jordan, A. M. The Medicinal Chemist's Toolbox: An Analysis of Reactions Used in the Pursuit of Drug Candidates. J. Med. Chem. 2011, 54 (10), 3451-3479. https://doi.org/10.1021/jm200187y.

(18) Declerck, V.; Nun, P.; Martinez, J.; Lamaty, F. Solvent-Free Synthesis of Peptides. Angew. Chemie Int. Ed. 2009,48 (49), $9318-9321$. https://doi.org/10.1002/anie.200903510.

(19) Métro, T.-X.; Bonnamour, J.; Reidon, T.; Sarpoulet, J.; Martinez, J.; Lamaty, F. Mechanosynthesis of Amides in the Total Absence of Organic Solvent from Reaction to Product Recovery. Chem. Commun. 2012, 48 (96), 11781-11783. https://doi.org/10.1039/C2CC36352F.

(20) Štrukil, V.; Bartolec, B.; Portada, T.; Đilović, I.; Halasz, I.; Margetić, D. One-Pot Mechanosynthesis of Aromatic Amides and Dipeptides from Carboxylic Acids and Amines. Chem. Commun. 2012, 48 (99), 12100-12102. https://doi.org/10.1039/C2CC36613D.

(21) Gonnet, L.; Tintillier, T.; Venturini, N.; Konnert, L.; Hernandez, J.-F.; Lamaty, F.; Laconde, G.; Martinez, J.; Colacino, E. N-Acyl Benzotriazole Derivatives for the Synthesis of Dipeptides and Tripeptides and Peptide Biotinylation by Mechanochemistry. ACS Sustain. Chem. Eng. 2017, 5 (4), 2936-2941. https://doi.org/10.1021/acssuschemeng.6b02439.

(22) Ardila-Fierro, K. J.; Crawford, D. E.; Körner, A.; James, S. L.; Bolm, C.; Hernández, J. G. Papain-Catalysed Mechanochemical Synthesis of Oligopeptides by Milling and Twin-Screw Extrusion: Application in the Juliá-Colonna Enantioselective Epoxidation. Green Chem. 2018, 20 (6), 1262-1269. https://doi.org/10.1039/C7GC03205F.

(23) Nicholson, W. I.; Barreteau, F.; Leitch, J. A.; Payne, R.; Priestley, I.; Godineau, E.; Battilocchio, C.; Browne, D. L. Direct Amidation of Esters by Ball Milling**. Angew. Chemie Int. Ed. 2021, 60 (40), 21868-21874. https://doi.org/https://doi.org/10.1002/anie.202106412.

(24) Margetić, D.; Štrukil, V. Chapter 3 - Carbon-Nitrogen Bond-Formation Reactions; Margetić, D., Štrukil, V. B. T.-M. O. S., Eds.; Elsevier: Boston, 2016; pp 141-233. https://doi.org/https://doi.org/10.1016/B978-0-12-802184-2.00003-0.

(25) Nun, P.; Martin, C.; Martinez, J.; Lamaty, F. Solvent-Free Synthesis of Hydrazones and Their Subsequent N-Alkylation in a Ball-Mill. Tetrahedron 2011, 67 (42), 8187-8194. https://doi.org/https://doi.org/10.1016/j.tet.2011.07.056.

(26) Métro, T.-X.; Salom-Roig, X. J.; Reverte, M.; Martinez, J.; Lamaty, F. Faster and Cleaner Dynamic Kinetic Resolution via Mechanochemistry. Green Chem. 2015, 17 (1), 204-208. https://doi.org/10.1039/C4GC01416B.

(27) Briš, A.; Đud, M.; Margetić, D. Mechanochemical N-Alkylation of Imides. Beilstein J. Org. Chem. 2017, 13, 1745-1752.

(28) Martina, K.; Rinaldi, L.; Baricco, F.; Boffa, L.; Cravotto, G. Highly Efficient Mechanochemical N-Arylation of Amino Alcohols and Diamines with Cu0 Powder. Synlett 2015, 26 (20), 2789-2794.

(29) Kubota, K.; Seo, T.; Koide, K.; Hasegawa, Y.; Ito, H. Olefin-Accelerated Solid-State C-N Cross-Coupling Reactions Using Mechanochemistry. Nat. Commun. 2019, 10 (1), 111. https://doi.org/10.1038/s41467-018-08017-9.

(30) Andersen, J. M.; Starbuck, H. F. Rate and Yield Enhancements in Nucleophilic Aromatic Substitution Reactions via Mechanochemistry. J. Org. Chem. 2021. https://doi.org/10.1021/acs.joc.0c02996.

(31) Lingome, C. E.; Pourceau, G.; Gobert-Deveaux, V.; Wadouachi, A. Efficient Synthesis of Glycosylamines in Solventless Conditions Promoted by Mechanical Milling. RSC Adv. 2014, 4 (68), 36350-36356. https://doi.org/10.1039/c4ra04321a.

(32) Wetzel, A.; Wöckel, S.; Schelwies, M.; Brinks, M. K.; Rominger, F.; Hofmann, P.; Limbach, M. Selective Alkylation of Amines with Alcohols by Cp*Iridium(III) Half-Sandwich Complexes. Org. Lett. 2013, 15 (2), 266-269. https://doi.org/10.1021/ol303075h.

(33) Watson, A. J. A.; Maxwell, A. C.; Williams, J. M. J. Borrowing Hydrogen Methodology for Amine Synthesis under Solvent-Free Microwave Conditions. J. Org. Chem. 2011, 76 (7), 2328-2331. https://doi.org/10.1021/jo102521a. 
the Key Role of Carboxylic Acid H-Bonding. European J. Org. Chem. 2011, 2011 (30), 5981-5990. https://doi.org/10.1002/ejoc.201100714.

(35) Zhang, G.; Yin, Z.; Zheng, S. Cobalt-Catalyzed N-Alkylation of Amines with Alcohols. Org. Lett. 2016,18 (2), $300-303$. https://doi.org/10.1021/acs.orglett.5b03461.

(36) Mastalir, M.; Tomsu, G.; Pittenauer, E.; Allmaier, G.; Kirchner, K. Co(II) PCP Pincer Complexes as Catalysts for the Alkylation of Aromatic Amines with Primary Alcohols. Org. Lett. 2016, 18 (14), 3462-3465. https://doi.org/10.1021/acs.orglett.6b01647.

Dalidovich, T.; Mishra, K. A.; Shalima, T.; Kudrjašova, M.; Kananovich, D. G.; Aav, R. Mechanochemical Synthesis of Amides with Uronium-Based Coupling Reagents: A Method for Hexa-Amidation of Biotin[6]Uril. ACS Sustain. Chem. Eng. 2020, 8 (41), $15703-15715$. https://doi.org/10.1021/acssuschemeng.0c05558.

(38) El-Faham, A.; Albericio, F. Morpholine-Based Immonium and Halogenoamidinium Salts as Coupling Reagents in Peptide Synthesis. J. Org. Chem. 2008, 73 (7), 2731-2737. https://doi.org/10.1021/jo702622c.

(39) El-Faham, A.; Albericio, F. COMU: A Third Generation of Uronium-Type Coupling Reagents. J. Pept. Sci. 2010,16 (1), 6-9. https://doi.org/10.1002/psc.1204.

(40) Poelert, M. A.; Kellogg, R. M.; Hulshof, L. A. Nucleophilic Substitutions Using O-alkyl-N,N'-dialkylisoureas. Applications to Ephedrines. Recl. des Trav. Chim. des Pays-Bas 1994, 113 (7-8), 365-368. https://doi.org/10.1002/recl.19941130705.

(41) Chighine, A.; Crosignani, S.; Arnal, M. C.; Bradley, M.; Linclau, B. Microwave-Assisted Ester Formation Using O-Alkylisoureas: A Convenient Method for the Synthesis of Esters with Inversion of Configuration. J. Org. Chem. 2009, 74 (13), 4753-4762. https://doi.org/10.1021/jo900476y.

(42) Mathias, L. J. Esterification and Alkylation Reactions Employing Isoureas. Synthesis (Stuttg). 1979, 1979 (08), 561-576.

(43) Sood, D. E.; Champion, S.; Dawson, D. M.; Chabbra, S.; Bode, B. E.; Sutherland, A.; Watson, A. J. B. Deoxyfluorination with CuF2: Enabled by Using a Lewis Base Activating Group. Angew. Chemie Int. Ed. 2020, 59 (22), 8460-8463. https://doi.org/https://doi.org/10.1002/anie.202001015.

(44) Li, Z.; Crosignani, S.; Linclau, B. A Mild, Phosphine-Free Method for the Conversion of Alcohols into Halides (Cl, $\mathrm{Br}$, I) via the Corresponding OAlkyl Isoureas. Tetrahedron Lett. 2003, 44 (44), 8143-8147. https://doi.org/10.1016/j.tetlet.2003.09.024.

(45) Merad, J.; Matyašovský, J.; Stopka, T.; Brutiu, B. R.; Pinto, A.; Drescher, M.; Maulide, N. Stable and Easily Available Sulfide Surrogates Allow a Stereoselective Activation of Alcohols. Chem. Sci. 2021, 12 (22), 7770-7774. https://doi.org/10.1039/d1sc01602d.

(46) Bellavance, G.; Dubé, P.; Nguyen, B. Tetramethylfluoroformamidinium Hexafluorophosphate (TFFH) as a Mild Deoxofluorination Reagent. Synlett 2012, 2012 (04), 569-574.

(47) Pittelkow, M.; Kamounah, F. S.; Boas, U.; Pedersen, B.; Christensen, J. B. TFFH as an Excellent Reagent for Acylation of Alcohols, Thiols and Dithiocarbamates. Synthesis (Stuttg). 2004, 2004 (15), 2485-2492.

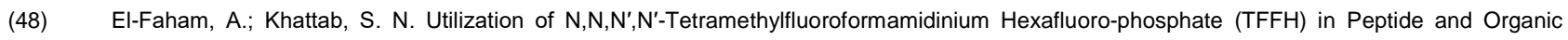
Synthesis. Synlett 2009, 2009 (06), 886-904.

(49) Barton, D. H. R.; Elliott, J. D.; Géro, S. D. The Synthesis and Properties of a Series of Strong but Hindered Organic Bases. J. Chem. Soc.\{,\} Chem. Commun. 1981, No. 21, 1136-1137. https://doi.org/10.1039/C39810001136.

(50) Friščić, T.; Childs, S. L.; Rizvi, S. A. A.; Jones, W. The Role of Solvent in Mechanochemical and Sonochemical Cocrystal Formation: A SolubilityBased Approach for Predicting Cocrystallisation Outcome. CrystEngComm 2009, 11 (3), 418-426. https://doi.org/10.1039/B815174A.

(51) Prat, D.; Hayler, J.; Wells, A. A Survey of Solvent Selection Guides. Green Chem. 2014, 16 (10), 4546-4551. https://doi.org/10.1039/C4GC01149J.

(52) Prat, D.; Wells, A.; Hayler, J.; Sneddon, H.; McElroy, C. R.; Abou-Shehada, S.; Dunn, P. J. CHEM21 Selection Guide of Classical- and Less Classical-Solvents. Green Chem. 2016, 18 (1), 288-296. https://doi.org/10.1039/C5GC01008J.

(53) Liang, C.; Behnam, M. A. M.; Sundermann, T. R.; Klein, C. D. Phenylglycine Racemization in Fmoc-Based Solid-Phase Peptide Synthesis: Stereochemical Stability is Achieved by Choice of Reaction Conditions. Tetrahedron Lett. 2017, 58 (24), $2325-2329$. https://doi.org/10.1016/j.tetlet.2017.04.047.

(54) General, I. Isomerizations. Org. Chem. Enzym. React. 2002, 359-397. https://doi.org/10.1016/b978-0-08-051336-2.50014-0.

(55) Bonnamour, J.; Métro, T.-X.; Martinez, J.; Lamaty, F. Environmentally Benign Peptide Synthesis Using Liquid-Assisted Ball-Milling: Application to 
the Synthesis of Leu-Enkephalin. Green Chem. 2013, 15 (5), 1116-1120. https://doi.org/10.1039/C3GC40302E.

(56) Druker, B. J.; Tamura, S.; Buchdunger, E.; Ohno, S.; Segal, G. M.; Fanning, S.; Zimmermann, J.; Lydon, N. B. Effects of a Selective Inhibitor of the Abl Tyrosine Kinase on the Growth of Bcr-Abl Positive Cells. Nat. Med. 1996, 2 (5), 561-566. https://doi.org/10.1038/nm0596-561.

Hahn, K. A.; Oglivie, G.; Rusk, T.; Devauchelle, P.; Leblanc, A.; Legendre, A.; Powers, B.; Leventhal, P. S.; Kinet, J.-P.; Palmerini, F.; Dubreuil, P.; Moussy, A.; Hermine, O. Masitinib Is Safe and Effective for the Treatment of Canine Mast Cell Tumors. J. Vet. Intern. Med. 2008, 22 (6), 13011309. https://doi.org/https://doi.org/10.1111/j.1939-1676.2008.0190.x.

(58) Suzuki, T.; Ando, T.; Tsuchiya, K.; Fukazawa, N.; Saito, A.; Mariko, Y.; Yamashita, T.; Nakanishi, O. Synthesis and Histone Deacetylase Inhibitory Activity of New Benzamide Derivatives. J. Med. Chem. 1999, 42 (15), 3001-3003. https://doi.org/10.1021/jm980565u.

(59) Letavic, M. A.; Aluisio, L.; Apodaca, R.; Bajpai, M.; Barbier, A. J.; Bonneville, A.; Bonaventure, P.; Carruthers, N. I.; Dugovic, C.; Fraser, I. C.; Kramer, M. L.; Lord, B.; Lovenberg, T. W.; Li, L. Y.; Ly, K. S.; Mcallister, H.; Mani, N. S.; Morton, K. L.; Ndifor, A.; Nepomuceno, S. D.; Pandit, C. R.; Sands, S. B.; Shah, C. R.; Shelton, J. E.; Snook, S. S.; Swanson, D. M.; Xiao, W. Novel Benzamide-Based Histamine H3 Receptor Antagonists: The Identification of Two Candidates for Clinical Development. ACS Med. Chem. Lett. 2015, 6 (4), 450-454. https://doi.org/10.1021/ml5005156.

(60) Vogel, P.; Figueira, S.; Muthukrishnan, S.; Mack, J. Environmentally Benign Nucleophilic Substitution Reactions. Tetrahedron Lett. 2009, 50 (1), 55-56. https://doi.org/https://doi.org/10.1016/j.tetlet.2008.10.079.

(61) Ortiz-Trankina, L. N.; Crain, J.; Williams, C.; Mack, J. Developing Benign Syntheses Using lon Pairs via Solvent-Free Mechanochemistry. Green Chem. 2020, 22 (11), 3638-3642. https://doi.org/10.1039/D0GC01116A.

(62) Leung, W.-P.; Chan, Y.-C. Alkali Metals: Inorganic Chemistry. Encycl. Inorg. Bioinorg. Chem. 2014, No. March 2014 , 1-26. https://doi.org/10.1002/9781119951438.eibc0003.pub2. 


\section{Entry for the Table of Contents}

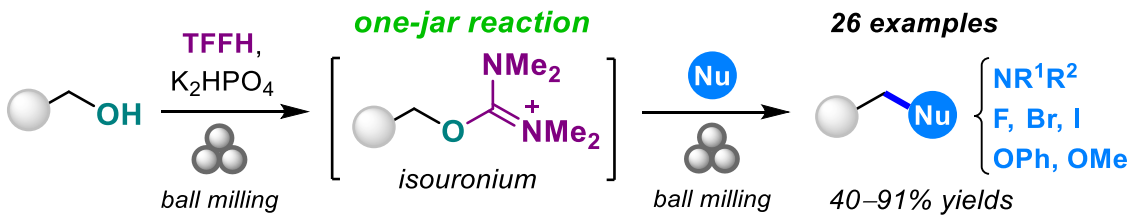

Towards greener amine synthesis: Nucleophilic substitution of alcohols via reactive isouronium salts under mechanochemical one-jar reaction conditions is presented for the first time. The method opens access towards biologically active amines, including active pharmaceutical ingredients.

Institute and/or researcher Twitter usernames: @TallinnTech @DzmitryKananov1 @RiinaAav 